\title{
Educando en competencias transversales a través de la asignatura de ingeniería de proyectos. Caso de estudio en el máster de Ingeniero de Minas. \\ Teaching transversal competences in the project management subject. Case of study: Master of Mining Engineering
}

\author{
Bernardo Llamas ${ }^{1}$, M. Dolores Storch de Gracia ${ }^{2}$, Ángel Cámara ${ }^{3}$ \\ bernardo.llamas@upm.es, lola.storch@upm.es, angel.camara@upm.es \\ ${ }^{1}$ Departamento Ingeniería Geológica y \\ Minera \\ ETS Ingenieros de Minas y Energía. \\ Universidad Politécnica de Madrid \\ Madrid, España \\ 2 Departamento Ingeniería Organización, \\ Administración de Empresas y \\ Estadística \\ ETS Ingenieros Industriales \\ Universidad Politécnica de Madrid \\ Madrid, España \\ ${ }^{3}$ Departamento Energía y \\ Combustibles \\ ETS Ingenieros de Minas y Energía. \\ Universidad Politécnica de Madrid \\ Madrid, España
}

Resumen- El contexto global y el acelerado cambio tecnológico en muchos de los sectores de la ingeniería obliga a que los futuros ingenieros deban aportar soluciones competitivas. En el presente trabajo se muestran resultados sobre competencias transversales como la creatividad, la comunicación y el trabajo en equipo. La creatividad es una de las competencias transversales más valoradas por el tejido industrial. Especial relevancia tiene los diferentes roles de un equipo y la importancia de uno de ellos para el éxito en el desarrollo de un proyecto. El caso de estudio que se presenta parte de un ejercicio realizado en el master de ingeniero de minas impartido por la Universidad Politécnica de Madrid, donde se han identificado roles clave para el desarrollo de proyectos innovadores desde una visión holística.

Palabras clave: competencias transversales; creatividad; comunicación; trabajo en equipo; master ingeniero de minas.

\begin{abstract}
The global context and accelerated technological change in many engineering sectors means that future engineers must provide competitive solutions. In the present work results are shown on transversal competences such as creativity, communication and teamwork. Creativity is one of the transversal competences most valued by the industrial fabric. Special relevance has the different roles of a team and the importance of one of them for success in developing a project. The case study is part of an exercise carried out in the master of mining engineer taught by the Polytechnic University of Madrid, where key roles have been identified for the development of innovative projects from a holistic view.
\end{abstract}

Keywords: transversal competences, creativity, communication, teamwork, MsD mining engineering.

\section{INTRODUCCIÓN}

La creatividad es la creación de un producto nuevo y útil (que aporte valor), a partir de ideas concretas. El concepto de creatividad se suele considerar multidimensional. La creatividad suele suponer el primer paso en el proceso de innovación, donde la creatividad genera el conjunto de ideas que permite posteriormente evaluar y seleccionar aquellas que sean apropiadas.

En el marco lógico de la gestión de un proyecto de investigación, este parte de las ideas innovadoras o creatividad del equipo de proyecto - a diferencia de un proceso, un proyecto siempre cuenta, en mayor o menor medida, con soluciones innovadoras.

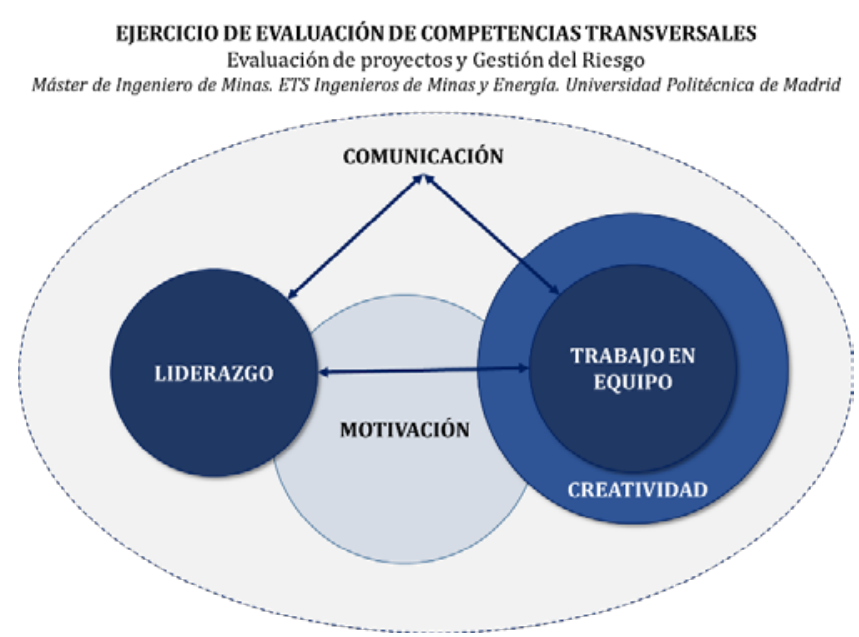

Figura 1. Relación de competencias trasversales evaluadas en la ingeniería de proyectos.

Pero, ¿cómo se fomenta la creatividad en un equipo? Buena parte de la responsabilidad recae en el estilo de liderazgo o el mal denominado jefe de proyecto. El estilo de liderazgo permitirá que los miembros del equipo de proyecto estén comprometidos con el éxito del proyecto $y$, por tanto, dispuestos a colaborar en un clima favorable y positivo a aportar sus ideas (creatividad). Pero, no solo será responsabilidad del coordinador del proyecto, sino que la cultura en la empresa será un entorno interno en el que el 
equipo deberá trabajar y, éste tendrá una influencia vital en el éxito del proyecto.

$\mathrm{Y}$, ¿el líder hace un equipo? La respuesta es que la suma de un grupo cohesionado es siempre más fructífera y productiva que un solo individuo, por muy brillante que este sea. Por tanto, la estrategia de configuración del equipo de trabajo y la distribución de roles serán piezas clave para la cohesión y trabajo en equipo.

Por tanto, el éxito de un proyecto, no solo depende de factores técnicos, sino que son más importantes competencias como: creatividad, trabajo en equipo, comunicación y liderazgo (Figura 1).

$\mathrm{Y}$, ¿cómo se educa en estas competencias en asignaturas como la ingeniería de proyectos? No existe un consenso o patrón establecido, y son muchos los estudios los que han valorado competencias, en un entorno docente, como la creatividad (tanto de una forma cuantitativa como cualitativa), la comunicación y el trabajo en equipo; mientras que son menos el número de estudios sobre liderazgo y estilos de liderazgo.

En el presente estudio se presenta una metodología de valoración de estas competencias basado en un enfoque práctico - desarrollo de un ejercicio y proyecto innovador. Para ello, se utilizarán técnicas de valoración de equipos (test Belbin), liderazgo (teoría Blanchard) y, por último, una valoración sobre creatividad y comunicación, mediante el pensamiento divergente para la creatividad y valoración por juicio de expertos para creatividad y comunicación.

\section{CONTEXTO}

La formación en ingeniería requiere de cada vez una formación sobre competencias transversales, de forma que el ingeniero no solo adquiera conocimientos propios de su área de especialización, sino que también adquiera una serie de competencias que faciliten la incorporación al mercado laboral.

El máster de Ingeniero de Minas, impartido por la Universidad Politécnica de Madrid, se encuentra acreditado por la prestigiosa institución ABET (Accreditation Board for Engineering and Technology, Inc www.abet.org). Esto hace que muchas de las competencias recogidas en la asignatura de “Evaluación de Proyectos y Gestión del Riesgo” estén recogidas dentro de las once competencias que esta institución considera esenciales en la formación de la ingeniería: aplica, experimenta, diseña, utiliza herramientas, resuelve y se actualiza - como competencias relacionadas con el conocimiento adquirido; es responsable, trabajo en equipo, comunica, valora y entiende los impactos y es contemporáneo como competencias transversales.

Mientras, otras competencias como liderazgo o creatividad son competencias explícitas recogidas entre las competencias propias de la citada Universidad y que así son recogidas en la ANECA (Agencia Nacional de Evaluación de la Calidad y Acreditación, www.aneca.es).

De entre las competencias analizadas en el presente trabajo, las competencias trabaja en equipo y comunica son reconocidas por ambas instituciones ABET y ANECA, mientras que lidera y creatividad son específicas por ANECA (y asumidas por la UPM).
Fruto del programa de mejora continua del máster, se trabaja en soluciones y herramientas docentes que permitan incrementar la formación del alumno en estas competencias.

\section{DESCRIPCIÓN}

El marco lógico de un proyecto de innovación se basa en dos aspectos fundamentales para el éxito del mismo: (i) la generación de una idea innovadora (solución novedosa y que aporte valor) y, (ii) la consecución o aprobación de la financiación para llevar ejecutar el proyecto. El éxito dependerá del desarrollo de las competencias transversales en las que se forma al alumno en la citada asignatura (Figura 2; Llamas \& Storch, 2017).

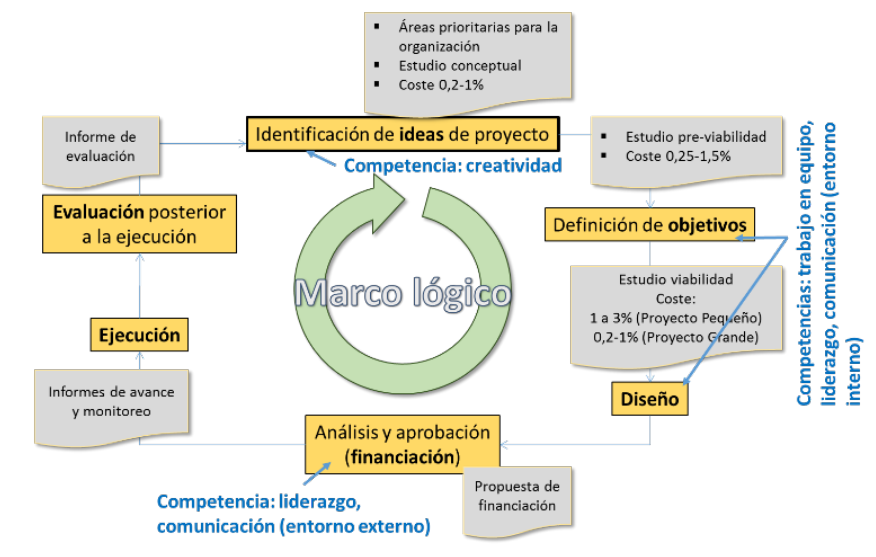

Figura 2. Marco Lógico de un proyecto de ingeniería.

Para ello, los alumnos deben resolver en equipo un proyecto de ingeniería - temática libre, pero asociado a sus áreas de conocimiento. Para ello, los alumnos realizarán el desarrollo del marco lógico de un proyecto, mediante la presentación de un análisis DAFO (Debilidades, Amenazas, Fortalezas y Oportunidades), análisis LEAN-CANVAS (desarrollo del modelo de negocio) y finalmente una ingeniería básica del proyecto.

En la evaluación continua de la asignatura, los alumnos adquieren el conocimiento deseado de los aspectos teóricos y su aplicación mediante los ejercicios arriba mencionados. La valoración final de la asignatura recoge estas valoraciones, tanto individual como de grupo, mediante la exposición de dichos trabajos y finalmente el trabajo y exposición final (ejercicio del inversionista).

La metodología de aprendizaje de la asignatura y valoración sobre adquisición de competencias se basa en dos aspectos diferenciados:

a. Valoración por parte de un juicio de expertos, ejercicio considerado para las competencias de creatividad y comunicación.

b. Valoración, mediante cuestionario, de las competencias de trabajo en equipo y liderazgo. En este caso, mediante cuestionario Belbin y cuestionarios sobre liderazgo situacional y teoría Jung.

\section{A. Creatividad. El ejercicio del inversionista}

La creatividad considerada como el paso inicial dentro del proceso de innovación, se considera una competencia clave para los futuros ingenieros (Denson et al, 2009). En este caso, 
la valoración de la creatividad, se basa en los principios de novedad y funcionalidad (técnica, económica y social) e impacto.

Si bien existen diferentes estudios sobre la valoración de la creatividad, tanto de forma cuantitativa como cualitativa, en este caso, se considera la valoración cuantitativa mediante la metodología de pensamiento divergente y la valoración cualitativa mediante juicio de expertos (Baer et al, 2009).

Con el fin de darle una mayor rigurosidad, se ha incorporado adicionalmente a la valoración del cuestionario un ejercicio novedoso: el ejercicio del inversionista, donde los expertos no solo valoran en una escala de Lickert las subcompetencias, sino que valoran y asignan un valor de inversión en función de la idea (Llamas et al, forthcoming).

\section{B. Liderazgo.}

La elección del coordinador de un proyecto es una de las decisiones más relevantes e importantes en la consecución del éxito de un proyecto (Cohen et al, 2013). Este término se hace más evidente cuanta más innovación requiere el proyecto, puesto que este requiere de la creatividad del equipo de trabajo.

La teoría del liderazgo situacional, enunciada por Hersey y Blanchard es una de las más reconocidas en el mundo empresarial (McGrath y Bates, 2014). Su reconocimiento ha venido por el mercado, frente a un programa de investigación académica rigurosa.

Esta teoría se basa en la necesidad de liderar equipos de trabajo e individuos heterogéneos. Lo que supone adaptar el estilo de coordinación de acuerdo a dos ejes: tareas (indicando instrucciones sobre cómo hacer el trabajo) y relaciones (alentando y apoyando personalmente al individuo). De esta forma, se pueden establecer cuatro estrategias de dirección: coaching, dirección, apoyo y delegación. Con el fin de ilustrar esta teoría, por ejemplo, en el extremo del eje tareas se sitúa el individuo focalizado en realizar el trabajo, abandonado las relaciones sociales o el trabajo en equipo. Por el contrario, en el extremo de relaciones nos encontramos al individuo que fomenta el trabajo en equipo y basa el desarrollo de sus tareas en base a estas relaciones sociales.

Aún más, la teoría de Jung (Jung, 1990), cuestiona cómo el individuo se enfrenta al trabajo, considerando aspectos psicológicos como la introversión/extroversión y la racionalidad. La valoración de esta competencia se ha llevado a cabo mediante cuestionarios específicos.

\section{Trabajo en equipo. Belbin}

Una de las competencias más valorada por el mercado es la capacidad del individuo para integrarse y cooperar en un equipo de trabajo. Y es que la figura organizativa en la empresa se basa en la integración de individuos en equipos de trabajo.

La teoría de Belbin (Belbin, 2010), tras siete años de estudio del comportamiento individual en equipos de trabajo, se basa en la definición de hasta nueve roles de equipo agrupados mediante las principales características: (i) roles de reflexión (cerebro, monitor, especialista), (ii) roles de acción (impulsor, implementador y finalizador) y (iii) roles sociales (cohesionador, coordinador, investigador de recursos). El éxito de los equipos de trabajo se basa en la integración de individuos que aporten al equipo roles diferenciados - un individuo puede tomar no solo un rol en un equipo - y que el conjunto de roles individuales signifique un equipo equilibrado en cuanto a roles representados.

La actitud o rol que tome un individuo en un equipo, no solo vendrá determinado por las características individuales de este, sino que también estará condicionado por el estado en el que se encuentre el equipo (teoría Tuckman) (McGrath y Bates, 2014). La valoración de esta competencia, se realiza mediante cuestionario específico.

\section{Comunicación}

Según el Project Management Institute $\left(\mathrm{PMI}^{\circledR}\right)$, el coordinador de un proyecto debe dedicar un $90 \%$ de su tiempo a comunicar, bien sea hacia el entorno interior (recabar apoyos, comunicar con sus colaboradores) y el entorno exterior (proveedores, cliente, administraciones y otros grupos de interés) (Llamas et al, 2016).

Para evaluar esta competencia, los expertos completaron un cuestionario específico donde se valoran diferentes indicadores de comunicación.

\section{Resultados}

El ejercicio innovador de valoración de competencias, permite establecer una metodología holística y completa sobre cuatro de las competencias transversales más relevantes para el estudiante de ingeniería. El caso de estudio aquí presentado podría ser reproducido en otras ramas educativas en ingeniería.

\section{A. Creatividad}

Los resultados obtenidos de la competencia creatividad se han basado en el estudio y realización de un ejercicio novedoso, basado en la práctica: el ejercicio del inversionista (B. Llamas et al, forthcoming).

La valoración por parte de seis expertos - tanto del sector privado como público y educación - indican un razonable valor para la creatividad - como aspecto innovador. Sin embargo, la funcionalidad se ve resentida por un valor inferior al valor medio de la escala Likert utilizada (escala de cinco valores, Figura 3).

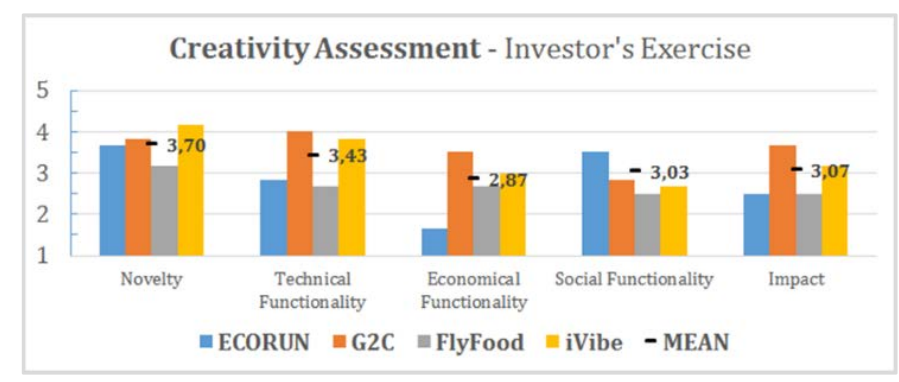

Figura 3. Evaluación de la creatividad para cinco equipos, según juicio de expertos.

\section{B. Liderazgo}

En cuanto a la valoración de la competencia liderazgo, como se ha recogido en el apartado anterior, se han analizado los estilos de liderazgo y su eficiencia de acuerdo a la teoría situacional. Los resultados mostrados en Figura 4 indican que los individuos asumen un liderazgo participativo (Q3) 
eficiente y muy acentuado en todos los individuos y grupos encuestados.

En menor proporción los individuos asumen un liderazgo instructivo (Q2) y basado en "dirigir" a los colaboradores (Q1). Es destacable, por el contrario, la baja puntuación obtenida en cuanto al estilo de dirección basado en la delegación (Q4).

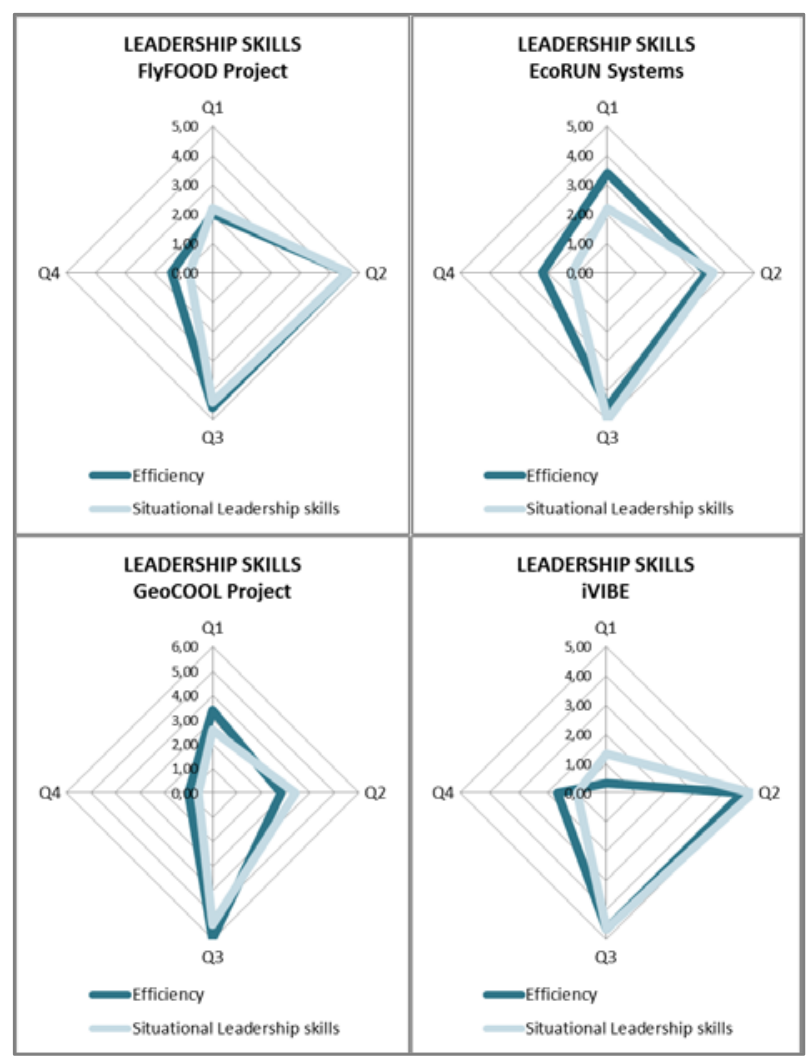

Figura 4. Resultados obtenidos de Estilo de liderazgo para cuatro equipos según teoría de liderazgo situacional.

El resultado obtenido de las encuestas específicas que analizan cómo el individuo se enfrenta al trabajo (teoría Jung).

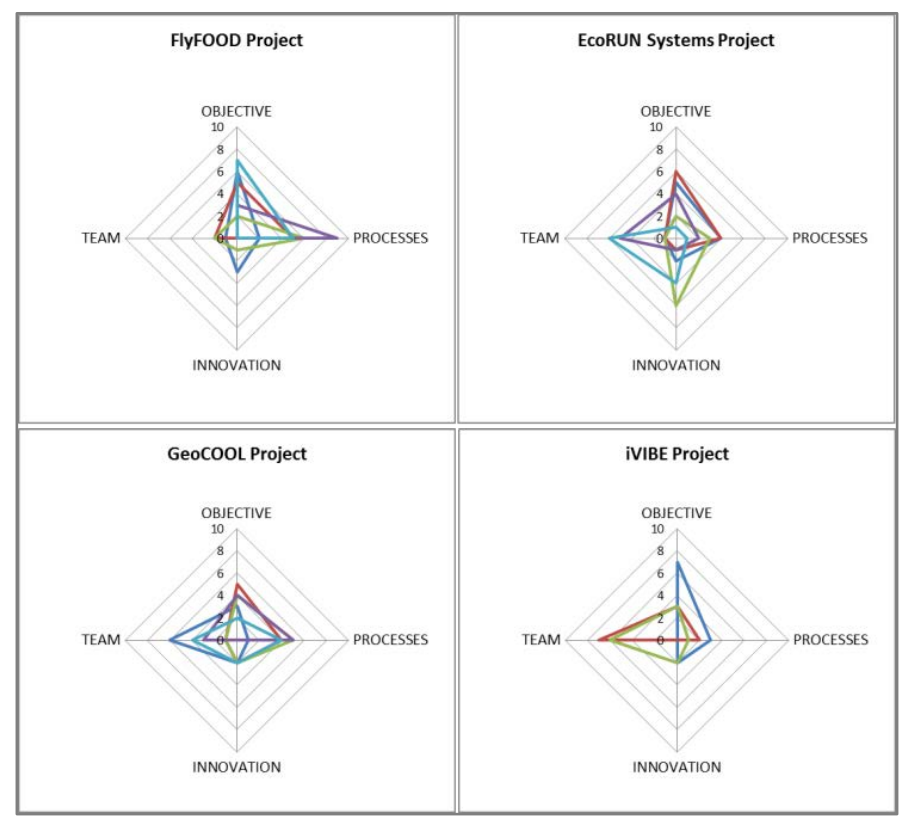

Figura 5. Resultados obtenidos de Actitud ante el trabajo para cuatro equipos según teoría Jung.

Es significativo el bajo nivel para el perfil de innovación (ver igualmente como este valor se confirma con el análisis de roles) para todos los equipos e individuos encuestados.

Los individuos sí tienen un perfil focalizado en objetivos y los procesos, y en menor medida en el equipo. Aunque este análisis del cuestionario se recoge en el análisis de liderazgo, este análisis está íntimamente relacionado con la competencia trabajo en equipo.

\section{Trabajo en equipo}

La competencia trabajo en equipo, en esta ocasión se ha analizado considerando los roles y los resultados alcanzados como equipo (ejercicio del inversionista).

En este caso, se valora el comportamiento del grupo (dinámica, comunicación, gestión de conflictos y otros, a través del cuestionario de Belbin).

Esta teoría describe el comportamiento del individuo en un equipo de trabajo mediante la definición de nueve roles, y un equipo será tanto más efectivo cuanto más balanceado estén los roles.

Un rol está definido por seis factores: personalidad, habilidad mental, actuales valores y motivaciones, limitaciones, experiencia y aprendizaje del rol.

Como se aprecia en la Figura 6, la mayoría de los equipos cuentan con un déficit en algunos de los roles fundamentales en la ingeniería de proyectos: el rol de la creatividad no se encuentra presente en la mayoría de los individuos. Pero igualmente es notorio la falta del rol "investigador de recursos", faceta fundamental en el proceso de consecución de financiación - este rol es especialista en comunicar con el exterior y buscar recursos para llevar a cabo el proyecto, conseguir los apoyos necesarios, etc.

\section{Comunicación}

La valoración de la competencia comunicación se llevó a cabo mediante cuestionario a expertos, desglosando la misma tanto escrita como oral (Tabla 1 ).

Tabla 1. Valoración de la competencia comunicación.

\begin{tabular}{|l|l|r|r|r|r||}
\hline & & ECORUN & G2C & FLYFOOD & iVIBE \\
\hline Estructura de la comunicación & Escrito & 3,3 & 3,2 & 4,3 & 3,7 \\
\hline Estilo & E & 2,8 & 2,8 & 3,8 & 3,5 \\
\hline Recursos necesarios & $\mathrm{E}$ & 3,0 & 2,8 & 3,7 & 3,3 \\
\hline Extensión & $\mathrm{E}$ & 3,3 & 3,3 & 3,3 & 3,8 \\
\hline Normas gramaticales & $\mathrm{E}$ & 3,5 & 3,3 & 4,0 & 3,2 \\
\hline Estructura de la comunicación & Oral & 3,3 & 3,0 & 3,8 & 3,2 \\
\hline Estilo & 0 & 2,8 & 3,2 & 3,7 & 3,2 \\
\hline Recursos necesarios & 0 & 3,2 & 3,3 & 3,3 & 3,3 \\
\hline Gestión del tiempo & 0 & 3,5 & 3,8 & 3,5 & 3,5 \\
\hline Técnicas de comunicación oral & 0 & 2,8 & 3,3 & 3,7 & 3,5 \\
\hline Escucha al interlocutor y responde & 0 & 3,2 & 3,8 & 4,2 & 4,0 \\
\hline \hline
\end{tabular}

Los equipos, en líneas generales presentan una valoración alta de esta competencia, demostrando que son capaces de exponer trabajos técnicos ante una serie de expertos, y con una escucha activa ante preguntas de los interlocutores.

Destaca el proyecto Flyfood con una alta valoración en esta competencia, que se constata y correlaciona con el mayor 
valor del equipo en cuanto al rol de "investigador de recursos" definido por Belbin.

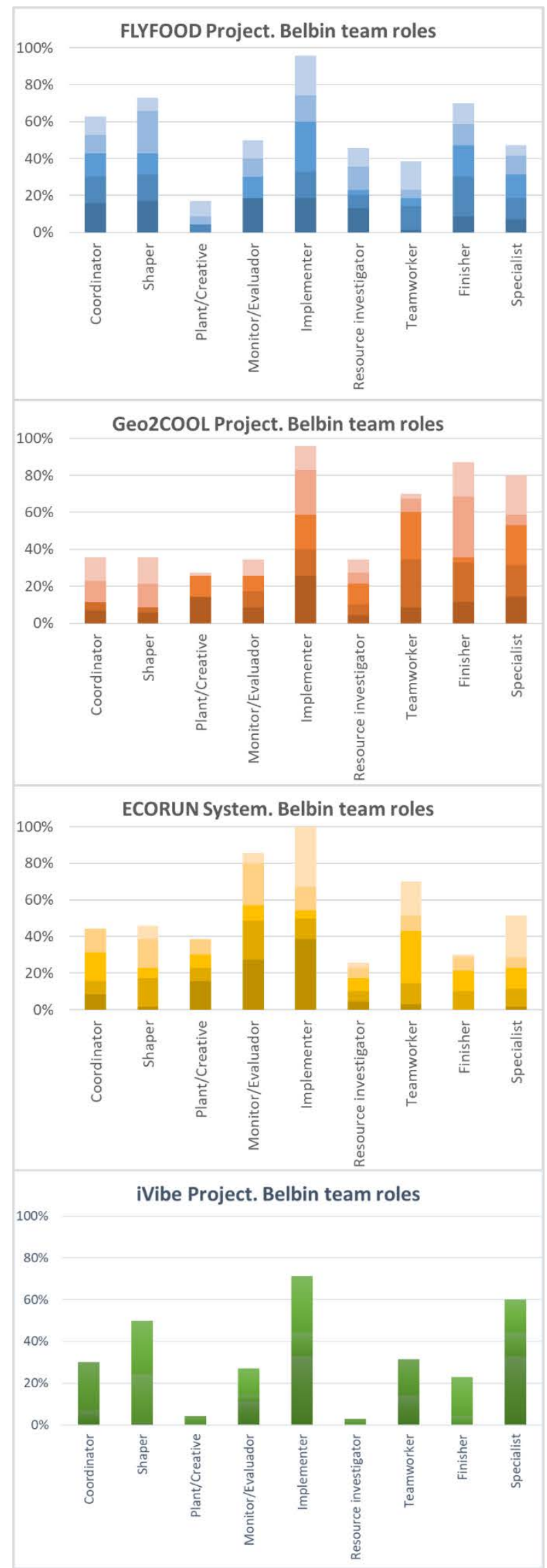

Figura 6. Resultados obtenidos de Roles presentes para cuatro equipos según teoría y cuestionario Belbin.

\section{CONCLUSIONES}

La valoración de competencias transversales como las aquí recogidas se antojan fundamentales en el contexto formativo de los futuros ingenieros de minas.

En este sentido, y recogiendo la filosofía de acercamiento de la empresa a la universidad, se considera razonable someter a los estudiantes a una valoración de competencias mediante el desarrollo del ejercicio del inversionista, donde se valoran aspectos objetivos y subjetivos de competencias como la creatividad y/o la comunicación.

La realización del cuestionario Belbin para que los estudiantes de máster conozcan en la práctica los roles que los miembros de un equipo pueden asumir, facilitará su integración en equipos de trabajo y la asunción de roles en el mismo.

Por último, el conocerse a uno mismo y cómo enfrenta cada individuo las relaciones laborales (cuestionario Jung), junto con el cuestionario sobre liderazgo situacional (cuestionario Blanchard) ahonda en las competencias sobre liderazgo.

Existe una correlación directa entre los tres cuestionarios realizados (teorías Blanchard, Belbin y Jung), y los resultados alcanzados en el ejercicio del inversionista, habiéndose demostrado la criticidad del "investigador de recursos" como rol clave en la consecución exitosa del proceso de financiación de un proyecto.

El ejercicio del inversionista recoge la mayoría de las competencias transversales consideradas más relevantes por los autores para el desempeño de la ingeniería de proyectos.

Esta metodología educativa, podrá aplicarse en contextos formativos de máster de ingeniería, donde se busca una formación especializada y una, cada vez más, cercanía con el mercado.

La aplicación de la misma debería completarse con el crucial estudio de la creación de equipos de trabajo. El análisis de equipos de trabajo equilibrados en cuanto a sus roles, y cómo el individuo afronta el trabajo y las relaciones que este conlleva, deberá ser considerado para el éxito en un área como la ingeniería de proyectos.

Por último, recoger que dos de los proyectos estudiados en la presente comunicación y, desarrollados por los alumnos en esta asignatura fueron presentados en la $14^{\text {a }}$ edición del concurso de ideas actuaupm (actúaupm, 2017). Ambos superaron la primera fase de valoración y los alumnos accedieron a una formación especializada sobre emprendimiento y empresa.

\section{REFERENCIAS}

ABET, Accreditation Board for Engineering and Technology, Inc http://www.abet.org/, acceso el 28 de mayo de 2017.

actúaupm, http://actuaupm.blogspot.com.es/, acceso el 04 de junio de 2017.

Agencia Nacional de Evaluación de la Calidad y Acreditación, ANECA, www.aneca.es, acceso el 28 de mayo de 2017. 
Baer J., McKool S.S., (2009). Assessing Creativity Using the Consensual Assessment Technique. In Handbook of Research on Assessment Technologies, Methods, and Applications in Higher Education, edited by C. Schreiner, 65-77. PA: IGI Global. doi:10.4018/978-160566-667-9.ch004.

Belbin R.M. (2010). Management Teams - Why they succeed or fail, Third edition, Elsevier. Oxford, United Kingdom.

Cohen Y., Keren B., Ornoy H., (2013). MBTI Personality Types of Project Managers and Their Success: A Field Survey. Project Management Journal, Vol. 44 (3), 7887.

Denson C.D., Lammi M.D., D’Amico S., (2015). Developing Instrumentation for Assessing Creativity in Engineering Design. Journal of Technology Education, 27(1), 23-40.

Jung C.G. (1990). Psychological types. Princeton, NJ: Princeton University Press.
Llamas B., Pous J., Storch de Gracia M.D. (2016). Apuntes de la asignatura de ingeniería de proyectos. Madrid, España Fundación Gómez-Pardo. ISBN: 978-84-6088312-8.

Llamas B., Storch de Gracia M.D. (2017). Apuntes de la asignatura de evaluación de proyectos y gestión del riesgo. De una idea a un proyecto. Madrid, España Fundación Gómez-Pardo. ISBN: 78-84-617-7988-8.

Llamas B., Storch de Gracia M.D., Mazadiego L.F., Pous J., Alonso J. (2017). Assessing creativity as a critical competence in engineering. Case of study in mines engineering degree. Revista de educación. Forthcoming.

McGrath J., Bates B, (2014) El pequeño libro de las grandes teorías del management. ALIENTA. ISBN: 9788415678908. 